\title{
GROUND STATES OF ELLIPTIC PROBLEMS INVOLVING NON HOMOGENEOUS OPERATORS
}

\author{
GIOVANY M. FIGUEIREDO AND HUMBERTO RAMOS QUOIRIN
}

\begin{abstract}
We investigate the existence of ground states for functionals with nonhomogenous principal part. Roughly speaking, we show that the Nehari manifold method requires no homogeinity on the principal part of a functional. This result is motivated by some elliptic problems involving nonhomogeneous operators. As an application, we prove the existence of a ground state and infinitely many solutions for three classes of boundary value problems.
\end{abstract}

\section{Introduction AND MAIN RESUlts}

This article is concerned with a class of variational elliptic problems involving non-homogeneous operators. Our main goal is to provide a unified approach to obtain ground state solutions for these problems. This approach is based on the Nehari manifold method, which was introduced in [28] and is by now a wellestablished and useful tool in finding solutions of problems with a variational structure, cf. 1, 4, 6, 7, 10, 11, 12, 13, 16, 31. In an abstract setting, given a Banach space $X$ and a $\mathcal{C}^{1}$ functional $\Phi: X \rightarrow \mathbb{R}$, a ground state of $\Phi$ is a solution $u_{0}$ of the problem

$$
\Phi^{\prime}\left(u_{0}\right)=0, \quad \Phi\left(u_{0}\right)=\min \{\Phi(u) ; u \text { is a critical point of } \Phi\} .
$$

When looking for such a solution, one may restrict $\Phi$ to the set

$$
\mathcal{N}=\left\{u \in X \backslash\{0\} ; \Phi^{\prime}(u) u=0\right\},
$$

which not only contains all nontrivial critical points of $\Phi$, but also turns out to have some useful properties. It is well-known that under some conditions on $\Phi, \mathcal{N}$ is a $\mathcal{C}^{1}$ submanifold of $X$ and critical points of the restriction of $\Phi$ to $\mathcal{N}$ are in fact critical points of $\Phi$. As an immediate consequence, one may obtain a ground state of $\Phi$ by minimizing $\Phi$ over $\mathcal{N}$. An overview of this procedure, as well as further developments and several applications of this method, are given in [30.

Throughout this article we assume that $\Omega \subset \mathbb{R}^{N}(N \geq 3)$ is a bounded domain and $f: \mathbb{R} \rightarrow[0, \infty)$ is an odd $\mathcal{C}^{1}$ function, so that $F(t)=\int_{0}^{t} f(s) d s$, defined for $t \in \mathbb{R}$, is an even function. A typical application of the Nehari manifold method provides the existence of a ground state for the prototype problem

$$
-\Delta u=f(u), \quad u \in H_{0}^{1}(\Omega), \quad u \geq 0,
$$

1991 Mathematics Subject Classification. 35J20, 35J25, 35J60, 35J92.

Key words and phrases. nonhomogeneous operator, variational methods, ground state, Nehari manifold.

The first author was supported by CNPq/PQ 301242/2011-9. The second author was supported by FONDECYT 11121567 . 
where $\Delta$ is the Laplace operator and $f$ is, in addition, subcritical and superlinear. More precisely, $f$ satisfies

$$
|f(s)| \leq C\left(1+|s|^{r-1}\right) \quad \forall s \in \mathbb{R}
$$

for some $C>0$ and $r \in\left(2,2^{*}\right)$, where $2^{*}=\frac{2 N}{N-2}$, as well as

$$
\lim _{s \rightarrow 0} \frac{f(s)}{s}=0, \quad \lim _{s \rightarrow \infty} \frac{f(s)}{s}=\infty, \quad \text { and } \quad \frac{f(s)}{s} \text { is increasing in }(0, \infty) .
$$

Then the functional

$$
\Phi(u):=\frac{1}{2}\|u\|^{2}-\int_{\Omega} F(u),
$$

defined on $H_{0}^{1}(\Omega)$, has a non-negative and nontrivial ground state $u_{0}$, which is a classical solution of (1.1). This result has a natural extension to an abstract setting as follows:

Let $X$ be a uniformly convex Banach space and $\mathcal{S}$ be the unit sphere in $X$. Assume that $\|\cdot\|$ is a $\mathcal{C}^{1}$ functional on $X \backslash\{0\}$. Then the following result holds, cf. [30, Theorem 13]:

Theorem 1.1. 30 Let $\Phi$ be such that $\Phi(0)=0$ and $\Phi=I_{0}-I$ where $I_{0}, I$ are $\mathcal{C}^{1}$ functionals on $X$ satisfying, for some $p>1$ :

(1) $I^{\prime}(u)=o\left(\|u\|^{p-1}\right)$ as $u \rightarrow 0$.

(2) $s \mapsto \frac{I^{\prime}(s u)}{s^{p-1}}$ is strictly increasing in $(0, \infty)$ for every $u \neq 0$.

(3) $\frac{I(s u)}{s^{p}} \rightarrow \infty$ uniformly for $u$ on weakly compact subsets of $X \backslash\{0\}$ as $s \rightarrow \infty$.

(4) $I^{\prime}$ is completely continuous. i.e. if $u_{n} \rightarrow u$ in $X$ then $I^{\prime}\left(u_{n}\right) \rightarrow I^{\prime}(u)$ in $X^{\prime}$.

(5) $I_{0}$ is weakly lower semicontinuous, positively homogeneous of degree p, i.e. $I_{0}(s u)=s^{p} I_{0}(u)$, and satisfies

$$
C_{0}\|u\|^{p} \leq I_{0}(u) \leq C_{0}^{-1}\|u\|^{p}
$$

and

$$
\left(I_{0}^{\prime}(v)-I_{0}^{\prime}(w)\right)(v-w) \geq C_{1}\left(\|v\|^{p-1}-\|w\|^{p-1}\right)(\|v\|-\|w\|)
$$

for some $C_{0}, C_{1}>0$ and every $u, v \in X$.

Then the equation $\Phi^{\prime}(u)=0$ has a ground state solution. Moreover, if $\Phi$ is even then this equation has infinitely many pairs of solutions.

The above theorem is clearly motivated by problems involving the $p$-Laplace operator, namely,

$$
-\Delta_{p} u=f(u), \quad u \in W_{0}^{1, p}(\Omega),
$$

where $\Delta_{p} u=\operatorname{div}\left(|\nabla u|^{p-2} \nabla u\right)$ and $p>1$. In this case $X=W_{0}^{1, p}(\Omega)$ with $\|u\|=\left(\int_{\Omega}|\nabla u|^{p}\right)^{\frac{1}{p}}$ and $\Phi=I_{0}-I$, where

$$
I_{0}(u)=\frac{1}{p}\|u\|^{p} \quad \text { and } \quad I(u)=\int_{\Omega} F(u) .
$$

Under conditions similar to (1.2), it can be shown that $I$ satisfies the assumptions of Theorem 1.1 so that (1.4) has a ground state solution and infinitely many pairs of solutions.

The following geometrical properties of $\Phi$ are essential in the proof of Theorem 1.1 . 
(A2) For any $w \in X \backslash\{0\}$ the map $t \mapsto \Phi(t w)$, defined for $t>0$, has a unique critical point $t_{w}>0$ which satisfies $\Phi\left(t_{w} w\right)=\max _{t>0} \Phi(t w)$.

(A3) $t_{w}$ is uniformly bounded away from zero for $w \in \mathcal{S}$, i.e. there exists $\delta>0$ such that $t_{w} \geq \delta$ for every $w \in \mathcal{S}$. Moreover, $t_{w}$ is bounded from above for $w$ in a compact subset of $\mathcal{S}$, i.e. given a compact set $\mathcal{W} \subset \mathcal{S}$ there exists $C_{\mathcal{W}}>0$ such that $t_{w} \leq C_{\mathcal{W}}$ for every $w \in \mathcal{W}$.

These properties are used to show that $\mathcal{S}$ is homeomorphic to $\mathcal{N}$ through the projection $w \mapsto t_{w} w$ and that one may carry out a critical point theory on $\mathcal{N}$, cf. [30, Corollary 10]. We shall prove that (A2) and (A3) hold for a larger class of functionals, in particular, for $\Phi=I_{0}-I$, where $I_{0}$ is not positively homogeneous and $I(u)=\int_{\Omega} F(u)$. This situation is motivated by the following examples:

(1) $X=W_{0}^{1, p}(\Omega)$ and

$$
I_{0}(u)=\frac{1}{p} \int_{\Omega} A\left(|\nabla u|^{p}\right) .
$$

Here $A(s)=\int_{0}^{s} a(t) d t$ and $a:[0, \infty) \rightarrow[0, \infty)$ is $\mathcal{C}^{1}$ in $(0, \infty)$ and satisfies

$$
k_{0}\left(1+t^{\frac{q-p}{p}}\right) \leq a(t) \leq k_{1}\left(1+t^{\frac{q-p}{p}}\right) \quad \forall t>0,
$$

where $k_{0}, k_{1}$ are positive constants and $p \geq q>1$. The associated EulerLagrange equation is the quasilinear equation

$$
-\operatorname{div}\left(a\left(|\nabla u|^{p}\right)|\nabla u|^{p-2} \nabla u\right)=f(u), \quad u \in W_{0}^{1, p}(\Omega) .
$$

This class of operators contains the $p$-Laplacian $(a(t) \equiv 1)$, as well as the sum of the $p$-Laplacian and the $q$-Laplacian $\left(a(t)=1+t^{\frac{q-p}{p}}\right)$. Problems involving this class of operators have been investigated, for instance, in 9, 14, 15, 20, 24, 26.

(2) $X=H_{0}^{1}(\Omega)$ and

$$
I_{0}(u)=\frac{1}{2} \hat{M}\left(\|u\|^{2}\right),
$$

where $\|u\|=\left(\int_{\Omega}|\nabla u|^{2}\right)^{\frac{1}{2}}, \hat{M}(s)=\int_{0}^{s} M(t) d t$ and $M:[0, \infty) \rightarrow[0, \infty)$ is a continuous function. In this case, the corresponding Euler-Lagrange equation is the Kirchhoff type equation

$$
-M\left(\int_{\Omega}|\nabla u|^{2}\right) \Delta u=f(u), \quad u \in H_{0}^{1}(\Omega),
$$

which has been intensively investigated over the last years, specially for $M(t)=a t+b$, with $a, b>0$, cf. 2, 8, 19, 23, 25, 27.

We shall prove that this equation has a ground state for a larger class of $M$, which includes, for instance,

$$
M(t)=m_{0}+\ln (1+t)
$$

or

$$
M(t)=m_{0}+\sum_{i=1}^{k} b_{i} t^{\gamma_{i}}
$$


where $b_{i} \geq 0$ and $\gamma_{i} \in(0,1]$ for $i=1, \ldots, k$, with $b_{i}>0$ for at least one $i$.

(3) Let $\vec{p}=\left(p_{1}, p_{2}, \ldots, p_{n}\right)$ with $p_{i}>1$ for $i=1, \ldots, N$ and $\sum_{i=1}^{N} \frac{1}{p_{i}}>1$.

Let $X=\mathcal{D}_{0}^{1, \vec{p}}(\Omega)$ be the completion of $\mathcal{C}_{0}^{\infty}(\Omega)$ with respect to the norm $\|u\|=\sum_{i=1}^{N}\left\|\partial_{i} u\right\|_{p_{i}}$. We set, for $u \in X$,

$$
I_{0}(u)=\sum_{i=1}^{N} \frac{1}{p_{i}} \int_{\Omega}\left|\partial_{i} u\right|^{p_{i}} .
$$

The corresponding Euler-Lagrange equation is the anisotropic equation

$$
-\sum_{i=1}^{N} \partial_{i}\left(\left|\partial_{i} u\right|^{p_{i}-2} \partial_{i} u\right)=f(u), \quad u \in \mathcal{D}_{0}^{1, \vec{p}}(\Omega) .
$$

For results on this class of problems, we refer to [3, 17, 21, 22, 32] and references therein.

We shall establish an abstract result (in the same style as Theorem 1.1) which applies to the problems above. In this sense, we shall prove that the Nehari manifold method applies to problems with nonhomogenous operators. To prove that $\Phi$ has a ground state, we follow a strategy slightly different from 30, since we do not prove that $\Phi$ satisfies the Palais-Smale condition at the ground state level. In doing so, we also get rid of the condition (1.3) and the uniform convexity assumption on $X$. This approach, in a rather simple setting, can be found in [5]. Once we have proved that the infimum of $\Phi$ over $\mathcal{N}$ is achieved, we shall deduce that it is a critical value of $\Phi$ thanks to the results of [30], which apply to $\mathcal{C}^{1}$ functionals.

Finally, let us recall (as pointed out in [30]) that the Nehari manifold method also has the advantage of not requiring an Ambrosetti-Rabinowitz type condition on $f$, which is customary when dealing with the Palais-Smale condition.

We state now our main result:

Theorem 1.2. Let $X$ be a reflexive Banach space such that $\|\cdot\|$ is a $\mathcal{C}^{1}$ functional on $X \backslash\{0\}$ and $\Phi: X \rightarrow \mathbb{R}$ be a $\mathcal{C}^{1}$ functional such that $\Phi(0)=0$. In addition, we assume that there exist $p, r>1$ such that:

(1) $\liminf _{u \rightarrow 0} \frac{\Phi^{\prime}(u) u}{\|u\|^{r}}>0$

(2) For every $u \in X$ we have $\Phi(u) \geq C_{0}\|u\|^{r}-I(u)$ where $C_{0}>0$ and $I$ is a weakly continuous functional on $X$.

(3) $\lim _{t \rightarrow \infty} \frac{\Phi(t u)}{t^{p}}=-\infty$ uniformly for $u$ on weakly compact subsets of $X \backslash\{0\}$.

(4) For every $u \in X \backslash\{0\}$ the maps $t \mapsto \frac{\Phi^{\prime}(t u) u}{t^{p-1}}$ and $t \mapsto \Phi(t u)-\frac{1}{p} \Phi^{\prime}(t u) t u$ are decreasing and increasing in $(0, \infty)$, respectively.

(5) $u \mapsto \Phi^{\prime}(u) u$ and $u \mapsto \Phi(u)-\frac{1}{p} \Phi^{\prime}(u) u$ are weakly lower semicontinuous on $X$.

Then $c:=\inf _{\mathcal{N}} \Phi$ is positive and achieved by some $u_{0} \neq 0$, i.e. $\Phi$ has a nontrivial ground state at a positive level. If, in addition, $\Phi$ is even then we may choose $u_{0} \geq 0$. 
Proof. The proof is divided in two steps: first we show that $c$ is achieved, and then we use the results of [30] to prove that $c$ is a critical value of $\Phi$.

Step 1: $c$ is achieved

Given $u \in X \backslash\{0\}$, we set $\gamma_{u}(t)=\Phi(t u)$ for $t>0$. From (1) and (3) it is clear that $\gamma_{u}(t)>0$ for $t$ sufficiently small and $\gamma_{u}(t)<0$ for $t$ sufficiently large. Consequently $\gamma_{u}$ has a global maximum point $t_{u}>0$, which is a critical point of $\gamma_{u}$. Since $t^{1-p} \gamma_{u}^{\prime}(t)=t^{1-p} \Phi^{\prime}(t u) u$, from (4) we infer that $t \mapsto t^{1-p} \gamma_{u}^{\prime}(t)$ is decreasing. It follows that $\gamma_{u}^{\prime}$ vanishes exactly once, i.e. $t_{u}$ is the unique critical point of $\gamma_{u}$. In particular, there holds $\Phi(u)>0$ for every $u \in \mathcal{N}$, so that $c \geq 0$.

We claim that $\mathcal{N}$ is bounded away from zero. Indeed, if $\left(u_{n}\right) \subset \mathcal{N}$ with $u_{n} \rightarrow 0$ in $X$ then $\frac{\Phi^{\prime}\left(u_{n}\right) u_{n}}{\left\|u_{n}\right\|^{r}}=0$ for every $n$, which contradicts (1). Thus the claim is proved.

Let us prove now that if $\left(u_{n}\right) \subset \mathcal{N}$ is such that $\left(\Phi\left(u_{n}\right)\right)$ is bounded from above then $\left(u_{n}\right)$ is bounded and, up to a subsequence, $u_{n} \rightarrow u_{0}$ with $u_{0} \not \equiv 0$. Assume by contradiction that $\left(u_{n}\right) \subset \mathcal{N}$ is unbounded. Then we may assume that $\left\|u_{n}\right\| \rightarrow \infty$ and $v_{n} \rightarrow v_{0}$, where $v_{n}=\frac{u_{n}}{\left\|u_{n}\right\|}$. If $v_{0} \equiv 0$ then, since $t=1$ is the global maximum point of $\gamma_{u_{n}}$, we have, using (2),

$$
\Phi\left(u_{n}\right) \geq \Phi\left(t v_{n}\right) \geq C_{0} t^{r}-I\left(t v_{n}\right) \rightarrow C_{0} t^{r}-I(0), \quad \forall t>0 .
$$

This contradicts the fact that $\left(\Phi\left(u_{n}\right)\right)$ is bounded from above. Hence $v_{0} \not \equiv 0$ and consequently, by (3),

$$
\frac{\Phi\left(u_{n}\right)}{\left\|u_{n}\right\|^{p}}=\frac{\Phi\left(\left\|u_{n}\right\| v_{n}\right)}{\left\|u_{n}\right\|^{p}} \rightarrow-\infty
$$

which contradicts the fact that $\Phi\left(u_{n}\right)>0$ for every $n$. Therefore $\left(u_{n}\right)$ must be bounded and, up to a subsequence, $u_{n} \rightarrow u_{0}$. If $u_{0} \equiv 0$ then, repeating the argument used in the case $v_{0} \equiv 0$, we get

$$
\Phi\left(u_{n}\right) \geq \Phi\left(t u_{n}\right) \geq C_{0} t^{r}\left\|u_{n}\right\|^{r}-I\left(t u_{n}\right) \geq D_{0} t^{r}-I\left(t u_{n}\right) \rightarrow D_{0} t^{r}-I(0),
$$

where we used (2) and the fact that $\mathcal{N}$ is bounded away from zero. So we get another contradiction, which shows that $u_{0} \not \equiv 0$. In particular, if $\left(u_{n}\right)$ is a minimizing sequence for $c$ then we may assume that $u_{n} \rightarrow u_{0}$ with $u_{0} \not \equiv 0$. Let $t_{0}=t_{u_{0}}$, i.e. $t_{0} u_{0} \in \mathcal{N}$. From (5), we infer that

$$
\Phi^{\prime}\left(u_{0}\right) u_{0} \leq \liminf \Phi^{\prime}\left(u_{n}\right) u_{n}=0,
$$

and, as a consequence, $t_{0} \leq 1$. We claim that $t_{0}=1$. Indeed, if $t_{0}<1$ then, using (4) and (5), we get

$$
\begin{aligned}
c & \leq \Phi\left(t_{0} u_{0}\right)=\Phi\left(t_{0} u_{0}\right)-\frac{1}{p} \Phi^{\prime}\left(t_{0} u_{0}\right) t_{0} u_{0}<\Phi\left(u_{0}\right)-\frac{1}{p} \Phi^{\prime}\left(u_{0}\right) u_{0} \\
& \leq \liminf \left(\Phi\left(u_{n}\right)-\frac{1}{p} \Phi^{\prime}\left(u_{n}\right) u_{n}\right)=\lim \Phi\left(u_{n}\right)=c,
\end{aligned}
$$

which is a contradiction. Therefore $t_{0}=1, u_{0} \in \mathcal{N}$, and $\Phi\left(u_{0}\right)=c$. Finally, if $\Phi$ is even then $\Phi\left(u_{0}\right)=\Phi\left(\left|u_{0}\right|\right)$, so that $\left|u_{0}\right|$ achieves $c$.

Step 2: $c$ is a critical value of $\Phi$ 
From the previous step it is clear that $\Phi$ satisfies (A2) and (A3) from [30]. By [30, Corollary 10], we deduce that $c=\inf _{\mathcal{S}} \Psi$, where $\Psi$ is defined by

$$
\Psi(w)=\Phi\left(t_{w} w\right) \quad \text { for } w \in \mathcal{S} .
$$

Moreover $\Psi$ is a $\mathcal{C}^{1}$ functional on $\mathcal{S}$, which is a $\mathcal{C}^{1}$ submanifold of $X$, and $w$ is a critical point of $\Psi$ if and only if $t_{w} w$ is a critical point of $\Phi$. This proves that $c$ is a critical value of $\Phi$.

\section{Remark 1.1.}

(1) We may easily check that the proof of Theorem 1.2 still can be carried out if instead of (2), the following conditions hold:

(A) For every $u \in X$ we have $\Phi(u)=I_{0}(u)-I(u)$, where $I$ is weakly continuous and $I_{0}$ is such that $\lim _{t \rightarrow \infty} I_{0}(t u)=\infty$ uniformly for $u \in \mathcal{S}$.

(B) For every $u \in X$ we have $\Phi^{\prime}(u) u=J_{0}(u)-J(u)$, where $J$ is weakly continuous and $J_{0}$ is such that $J_{0}\left(u_{n}\right) \rightarrow 0$ if and only if $u_{n} \rightarrow 0$.

As a matter of fact, one may repeat (1.8) and use (A) to get a contradiction if $\left(u_{n}\right) \subset \mathcal{N}$ is such that $\left(\Phi\left(u_{n}\right)\right)$ is bounded from above and $v_{n}=\frac{u_{n}}{\left\|u_{n}\right\|} \rightarrow 0$. Moreover, if $\left(u_{n}\right) \subset \mathcal{N}$ and $u_{n} \rightarrow u_{0}$ then $u_{0} \not \equiv 0$. Indeed, if $u_{0} \equiv 0$ then, from $J_{0}\left(u_{n}\right)-J\left(u_{n}\right)=\Phi^{\prime}\left(u_{n}\right) u_{n}=0$ and the weak continuity of $J$ we deduce that $J_{0}\left(u_{n}\right) \rightarrow 0$, so that, by (B), $u_{n} \rightarrow 0$, which contradicts the fact that $\mathcal{N}$ is bounded away from zero. The rest of the proof holds without further modifications.

(2) If $\Phi$ is weakly lower semi-continuous then, instead of (4) and (5), one may require only that for every $u \in X \backslash\{0\}$ the map $t \mapsto \frac{\Phi^{\prime}(t u) u}{t^{p-1}}$ is decreasing in $(0, \infty)$. Note indeed that one may still obtain a minimizing sequence for $c$ such that $u_{n} \rightarrow u_{0}$ and $u_{0} \not \equiv 0$. From the weak lower semicontinuity of $\Phi$ and the fact that $\Phi\left(u_{n}\right)=\max _{t>0} \Phi\left(t u_{n}\right)$ we deduce that

$$
c \leq \Phi\left(t_{0} u_{0}\right) \leq \liminf \Phi\left(t_{0} u_{n}\right) \leq \liminf \Phi\left(u_{n}\right)=c,
$$

i.e. $c$ is achieved.

(3) Unlike [30, we don't make use of the Palais-Smale condition of $\Phi$ to show that $c$ is achieved. Indeed, note that the proof of Theorem 1.2 does not require the strong convergence of a minimizing sequence for $c$.

(4) From the proof of Theorem 1.2, we shall highlight the following result: if $\left(u_{n}\right) \subset \mathcal{N}$ is such that $\left(\Phi\left(u_{n}\right)\right)$ is bounded from above then $\left(u_{n}\right)$ is bounded.

Following [30, we say that $\Phi$ satisfies the Palais-Smale condition on $\mathcal{N}$ if any Palais-Smale sequence of $\Phi$ which is moreover in $\mathcal{N}$ contains a convergent subsequence.

Combining Theorem 1.2 above and Theorem 2 and Corollary 10 from [30, we get the following result: 
Corollary 1.1. Under the assumptions of Theorem 1.2, assume in addition that $\Phi$ is even and satisfies the Palais-Smale condition on $\mathcal{N}$. Then $\Phi$ has infinitely many pairs of critical points.

\section{Applications}

We apply now Theorem 1.2 and Corollary 1.1 to the equations (1.5), (1.6) and (1.7). Let us recall that $\Omega \subset \mathbb{R}^{N}(N \geq 3)$ is a bounded domain, $f: \mathbb{R} \rightarrow[0, \infty)$ is an odd $\mathcal{C}^{1}$ function and $F(t)=\int_{0}^{t} f(s) d s$, for $t \in \mathbb{R}$.

2.1. A quasilinear equation. We assume that $a:[0, \infty) \rightarrow[0, \infty)$ is $\mathcal{C}^{1}$ in $(0, \infty)$ and we set $A(t)=\int_{0}^{t} a(s) d s$ for $t \in \mathbb{R}$.

Corollary 2.1. Under the above assumptions on a, assume in addition that there exist $p \geq q>1$ such that:

(1) $k_{0}\left(1+t^{\frac{q-p}{p}}\right) \leq a(t) \leq k_{1}\left(1+t^{\frac{q-p}{p}}\right), \quad \forall t>0$, where $k_{0}, k_{1}$ are positive constants.

(2) a is non-increasing.

(3) $t \mapsto a\left(t^{p}\right) t^{p}$ and $t \mapsto A\left(t^{p}\right)-a\left(t^{p}\right) t^{p}$ are convex in $(0, \infty)$.

(4) $\lim _{t \rightarrow 0} \frac{f(t)}{t^{q-1}}=0$.

(5) $\lim _{t \rightarrow \infty} \frac{F(t)}{t^{p}}=\infty$.

(6) $\lim _{t \rightarrow \infty} \frac{f(t)}{t^{\alpha-1}}=0$ for some $\alpha \in\left(p, p^{*}\right)$.

(7) $t \mapsto \frac{f(t)}{t^{p-1}}$ is increasing on $(0, \infty)$.

Then (1.5) has a nontrivial and non-negative ground state.

Proof. First of all, note that $A(t)$ is well-defined in view of (1). Let $X=W_{0}^{1, p}(\Omega)$ with $\|u\|=\left(\int_{\Omega}|\nabla u|^{p}\right)^{\frac{1}{p}}$. We set, for $u \in X$,

$$
\Phi(u)=\frac{1}{p} \int_{\Omega} A\left(|\nabla u|^{p}\right)-\int_{\Omega} F(u) .
$$

From (1) we infer that

$$
k_{0}\left(t^{p}+t^{q}\right) \leq a\left(t^{p}\right) t^{p} \leq k_{1}\left(t^{p}+t^{q}\right), \quad \forall t>0
$$

and

$$
k_{0}\left(t^{p}+\frac{p}{q} t^{q}\right) \leq A\left(t^{p}\right) \leq k_{1}\left(t^{p}+\frac{p}{q} t^{q}\right), \quad \forall t>0 .
$$

On the other hand, from (4) and (6) we infer that for any $\varepsilon>0$ there exists $C_{\varepsilon}>0$ such that

$$
|f(t)| \leq \varepsilon|t|^{q-1}+C_{\varepsilon}|t|^{\alpha-1}, \quad \forall t \in \mathbb{R} .
$$

Since $1<q \leq p$, it follows that $\Phi$ is a $\mathcal{C}^{1}$ functional on $X$. From (2.12) and the continuity of the embeddings $X \subset L^{\alpha}(\Omega)$ and $W_{0}^{1, q}(\Omega) \subset L^{q}(\Omega)$, we infer that for any $\varepsilon>0$ there exists $C_{\varepsilon}>0$ such that

$$
\left|\int_{\Omega} f(u) u\right| \leq \varepsilon \int_{\Omega}|\nabla u|^{q}+C_{\varepsilon}\|u\|^{\alpha} \quad \forall u \in X .
$$


Taking $\varepsilon>0$ sufficiently small and using (2.10), we get

$$
\Phi^{\prime}(u) u \geq\left(k_{0}-\varepsilon\right) \int_{\Omega}|\nabla u|^{q}+k_{0}\|u\|^{p}-C_{\varepsilon}\|u\|^{\alpha} \quad \forall u \in X,
$$

and consequently

$$
\liminf _{\|u\| \rightarrow 0} \frac{\Phi^{\prime}(u) u}{\|u\|^{p}}>0
$$

From (2.11), note also that

$$
\Phi(u) \geq \frac{k_{0}}{p}\|u\|^{p}-\int_{\Omega} F(u)
$$

and by the compact Sobolev embedding $X \subset L^{\alpha}(\Omega)$, the functional $u \mapsto \int_{\Omega} F(u)$ is weakly continuous on $X$. Still from (2.11), we have

$$
\frac{\Phi(t u)}{t^{p}} \leq \frac{k_{1}}{q} t^{q-p} \int_{\Omega}|\nabla u|^{q}+\frac{k_{1}}{p}\|u\|^{p}-\int_{\Omega} \frac{F(t u)}{t^{p}} \rightarrow-\infty,
$$

uniformly for $u$ on weakly compact subsets of $X \backslash\{0\}$, by (5). From (2) and (7) we have that

$$
t \mapsto \frac{\Phi^{\prime}(t u) u}{t^{p-1}}=\int_{\Omega} a\left(t^{p}|\nabla u|^{p}\right)|\nabla u|^{p}-\int_{\Omega} \frac{f(t u)}{t^{p-1}} u
$$

is decreasing on $(0, \infty)$ for every $u \neq 0$. Furthermore, it is clear that

$$
t \mapsto A\left(t^{p}\right)-a\left(t^{p}\right) t^{p} \text { is non-decreasing in }(0, \infty) .
$$

On the other hand, (7) provides that

$$
t \mapsto \frac{1}{p} f(t) t-F(t) \text { is increasing in }(0, \infty) \text {. }
$$

Thus $t \mapsto \Phi(t u)-\frac{1}{p} \Phi^{\prime}(t u) t u$ is increasing in $(0, \infty)$ for every $u \neq 0$.

Finally, (3) yields that $u \mapsto \Phi^{\prime}(u) u$ and $u \mapsto \Phi(u)-\frac{1}{p} \Phi^{\prime}(u) u$ are weakly lower semi-continuous on $X$. Therefore Theorem 1.2 applies with $r=p$ and since $\Phi$ is even, we infer that $\Phi$ has a nontrivial and non-negative ground state.

Let $\Psi: X \rightarrow \mathbb{R}$ be a $\mathcal{C}^{1}$ functional. Recall that $\Psi^{\prime}$ belongs to the class $\left(S_{+}\right)$ condition if

$$
u_{n} \rightarrow u_{0} \text { in } X, \quad \limsup \Phi^{\prime}\left(u_{n}\right)\left(u_{n}-u_{0}\right) \leq 0 \quad \Longrightarrow \quad u_{n} \rightarrow u_{0} \text { in } X .
$$

Set $\Psi(u)=\int_{\Omega} A\left(|\nabla u|^{p}\right)$ for $u \in W_{0}^{1, p}(\Omega)$. It is known that if $t \mapsto A\left(t^{p}\right)$ is strictly convex and satisfies

$$
a_{1} t^{p}-b_{1} \leq A\left(t^{p}\right) \leq a_{2} t^{p}+b_{2}, \quad \forall t>0
$$

for some positive constants $a_{1}, a_{2}, b_{1}, b_{2}$, then $\Psi^{\prime}$ belongs to the class $\left(S_{+}\right)$(see [18] for a proof).

As a consequence of Remark 1.1.(4), we see that under the assumptions of Corollary 2.1 and the condition

$$
t \mapsto A\left(t^{p}\right) \text { is strictly convex in }(0, \infty)
$$

the functional $\Phi$ given by (2.9) satisfies the Palais-Smale condition on $\mathcal{N}$. We infer then the following result:

Corollary 2.2. Under the assumptions of Corollary 2.1, assume in addition that (2.13) holds. Then the problem (1.5) has infinitely many pairs of solutions. 
Remark 2.1. One may easily check that Corollaries 2.1 and 2.2 apply in particular to $a(t) \equiv 1$ and $a(t)=1+t^{\frac{q-p}{p}}$, with $p>q>1$, which correspond to the operators $-\Delta_{p}$ and $-\Delta_{p}-\Delta_{q}$, respectively.

2.2. A nonlocal equation. Let $N=3$, so that $2^{*}=6$. We assume that $M:[0, \infty) \rightarrow[0, \infty)$ is a $\mathcal{C}^{1}$ function and we set $\hat{M}(t)=\int_{0}^{t} M(s) d s$ for $t \in \mathbb{R}$.

Corollary 2.3. Under the above assumptions on $M$, assume in addition:

(1) $M$ is increasing and $M(0):=m_{0}>0$.

(2) $t \mapsto \frac{M(t)}{t}$ is decreasing.

(3) $\lim _{t \rightarrow 0} \frac{f(t)}{t}=0$.

(4) $\lim _{t \rightarrow \infty} \frac{F(t)}{t^{4}}=\infty$.

(5) $\lim _{t \rightarrow \infty} \frac{f(t)}{t^{\alpha-1}}=0$ for some $\alpha \in(4,6)$.

(6) $t \mapsto \frac{f(t)}{t^{3}}$ is increasing.

Then (1.6) has a nontrivial and non-negative ground state.

Proof. Let $X=H_{0}^{1}(\Omega)$ with $\|u\|=\left(\int_{\Omega}|\nabla u|^{2}\right)^{\frac{1}{2}}$. We set, for $u \in X$,

$$
\Phi(u)=\frac{1}{2} \hat{M}\left(\|u\|^{2}\right)-\int_{\Omega} F(u) .
$$

Note that $\|\cdot\|$ is a $\mathcal{C}^{1}$ functional on $X$, so that $u \mapsto \hat{M}\left(\|u\|^{2}\right)$ is $\mathcal{C}^{1}$ as well. From (3) and (5) we have that for every $\varepsilon>0$ there exists $C_{\varepsilon}>0$ such that

$$
|f(t)| \leq \varepsilon|t|+C_{\varepsilon}|t|^{\alpha-1}, \quad \forall t \in \mathbb{R}
$$

Thus $\Phi$ is a $\mathcal{C}^{1}$ functional. Using (1) and (2.15), we have

$$
\Phi^{\prime}(u) u=M\left(\|u\|^{2}\right)\|u\|^{2}-\int_{\Omega} f(u) u \geq m_{0}\|u\|^{2}-\int_{\Omega} f(u) u .
$$

From (2.15) and the continuous embedding $X \subset L^{\alpha}(\Omega)$, we get

$$
\left|\int_{\Omega} f(u) u\right| \leq \varepsilon\|u\|^{2}+C_{\varepsilon}\|u\|^{\alpha}, \quad \forall u \in X .
$$

Thus we have, for $u \in X$,

$$
\Phi^{\prime}(u) u \geq m_{0}\|u\|^{2}-\varepsilon\|u\|^{2}-C_{\varepsilon}\|u\|^{\alpha} .
$$

Taking $\varepsilon>0$ sufficiently small, we get

$$
\liminf _{\|u\| \rightarrow 0} \frac{\Phi^{\prime}(u) u}{\|u\|^{2}}>0
$$

Moreover, from (2) we infer that

$$
\hat{M}(t)=\int_{0}^{t} \frac{M(s)}{s} s d s \geq \frac{M(t)}{t} \int_{0}^{t} s d s=\frac{1}{2} M(t) t, \quad \forall t>0,
$$

and consequently

$$
\Phi(u) \geq \frac{m_{0}}{2}\|u\|^{2}-\int_{\Omega} F(u)
$$


By (2.15) and the compact embedding $X \subset L^{\alpha}(\Omega)$, the functional $u \mapsto \int_{\Omega} F(u)$ is weakly continuous on $X$. Now, from (2) we have $M(t) \leq M(1) t$ for $t \geq 1$, so that

$$
M(t) \leq M(1) t+C, \quad \forall t \geq 0
$$

for some constant $C>0$. Consequently we have, for $u \in X$,

$$
\Phi(u) \leq C_{1}\|u\|^{4}+C_{2}\|u\|^{2}-\int_{\Omega} F(u),
$$

for some $C_{1}, C_{2}>0$, so that, by (4), $\frac{\Phi(t u)}{t^{4}} \rightarrow-\infty$ uniformly on weakly compact subsets of $X \backslash\{0\}$. From (2) and (6), it follows that

$$
t \mapsto \frac{\Phi^{\prime}(t u) u}{t^{3}}=\frac{1}{t^{2}} M\left(t^{2}\|u\|^{2}\right)\|u\|^{2}-\int_{\Omega} \frac{f(t u)}{t^{3}} u
$$

is decreasing for every $u \neq 0$.

Furthermore, note that (2) yields $t M^{\prime}(t) \leq M(t)$ for any $t>0$, and consequently

$$
t \mapsto \frac{1}{2} \hat{M}(t)-\frac{1}{4} M(t) t \quad \text { is increasing. }
$$

Hence $t \mapsto \Phi(t u)-\frac{1}{4} \Phi^{\prime}(t u) t u$ is increasing in $(0, \infty)$ for every $u \neq 0$.

Finally, since $M$ and $t \mapsto \frac{1}{2} \hat{M}(t)-\frac{1}{4} M(t) t$ are increasing, the mappings

$$
u \mapsto M\left(\|u\|^{2}\right) \quad \text { and } \quad u \mapsto \frac{1}{2} \hat{M}\left(\|u\|^{2}\right)-\frac{1}{4} M\left(\|u\|^{2}\right)\|u\|^{2}
$$

are weakly lower semicontinuous on $X$. Therefore Theorem 1.2 applies with $r=2$ and $p=4$. Note also that $\Phi$ is even. The proof is now complete.

Corollary 2.4. Under the assumptions of Corollary 2.3, the problem (1.6) has infinitely many pairs of solutions.

Proof. Let $\left(u_{n}\right) \subset \mathcal{N}$ be a Palais-Smale sequence for $\Phi$, defined in (2.14). By Remark 1.1. $(4)$, we know that $\left(u_{n}\right)$ is bounded, so that, up to a subsequence, $u_{n} \rightarrow u_{0}$ in $X$. From (2.15), we know that

$$
\int_{\Omega} f\left(u_{n}\right)\left(u_{n}-u_{0}\right)=o(1) .
$$

Hence

$$
M\left(\left\|u_{n}\right\|^{2}\right) \int_{\Omega} \nabla u_{n} \nabla\left(u_{n}-u_{0}\right)=\Phi^{\prime}\left(u_{n}\right)\left(u_{n}-u_{0}\right)+o(1)=o(1) .
$$

Since $M$ is continuous and $M(t) \geq m_{0}>0$ for all $t \geq 0$, we infer that $M\left(\left\|u_{n}\right\|^{2}\right)$ is bounded and bounded away from zero, so that

$$
\int_{\Omega} \nabla u_{n} \nabla\left(u_{n}-u_{0}\right)=o(1)
$$

By the uniform convexity of $H_{0}^{1}(\Omega)$, we get $u_{n} \rightarrow u_{0}$.

Remark 2.2. Besides $M(t)=a t+b$, with $a, b>0$, Corollaries 2.3 and 2.4 apply also to $M(t)=m_{0}+\ln (1+t)$ and $M(t)=m_{0}+\sum_{i=1}^{k} b_{i} t^{\gamma_{i}}$, where $b_{i} \geq 0$ and $\gamma_{i} \in(0,1]$ for $i=1, \ldots, k$, with $b_{i}>0$ for at least one $i$. 
2.3. A anisotropic equation. Let $1<p_{1} \leq p_{2} \leq \ldots \leq p_{N}$ be such that $\sum_{i=1}^{N} \frac{1}{p_{i}}>1$ and $p_{N}<p^{*}$, where $p^{*}=\frac{N}{\left(\sum_{i=1}^{N} \frac{1}{p_{i}}\right)-1}$. If $r>1$, we denote by $\|v\|_{r}$ the norm of $v$ in $L^{r}(\Omega)$.

Corollary 2.5. Under the above assumptions, assume in addition:

(1) $\lim _{t \rightarrow 0^{+}} \frac{f(t)}{t^{p_{1}-1}}=0$.

(2) $\lim _{t \rightarrow \infty} \frac{F(t)}{t^{p}}=\infty$.

(3) $\lim _{t \rightarrow \infty} \frac{f(t)}{t^{\alpha-1}}=\infty$ for some $\alpha \in\left(p_{N}, p^{*}\right)$.

(4) $t \mapsto \frac{f(t)}{t^{p} N^{-1}}$ is increasing.

Then (1.7) has a nontrivial and non-negative ground state.

Proof. Let $X=\mathcal{D}_{0}^{1, \vec{p}}(\Omega)$ be the completion of $\mathcal{C}_{0}^{\infty}(\Omega)$ with respect to the norm $\|u\|=\sum_{i=1}^{N}\left\|\partial_{i} u\right\|_{p_{i}}$. It is known that $X$ is a reflexive Banach space which embedds continuously in $L^{q}(\Omega)$ if $q \in\left[1, p^{*}\right]$, and compactly if $q \in\left[1, p^{*}\right)$, cf [21].

We set, for $u \in X$,

$$
\Phi(u)=\sum_{i=1}^{N} \frac{1}{p_{i}} \int_{\Omega}\left|\partial_{i} u\right|^{p_{i}}-\int_{\Omega} F(u) .
$$

Note that if $\|u\| \leq 1$ then $\left\|\partial_{i} u\right\|_{p_{i}}<1$ for $i=1, . ., N$, and since $p_{i} \leq p_{N}$ we get $\left\|\partial_{i} u\right\|_{p_{i}}^{p_{i}} \geq\left\|\partial_{i} u\right\|_{p_{i}}^{p_{N}}$ for $i=1, . ., N$. Thus

$$
\sum_{i=1}^{N}\left\|\partial_{i} u\right\|_{p_{i}}^{p_{i}} \geq \sum_{i=1}^{N}\left\|\partial_{i} u\right\|_{p_{i}}^{p_{N}} \geq C\left(\sum_{i=1}^{N}\left\|\partial_{i} u\right\|_{p_{i}}\right)^{p_{N}}=C\|u\|^{p_{N}} .
$$

Using (1), (3) and the continuous embedding $X \subset L^{\alpha}(\Omega)$, we have that for any $\varepsilon>0$ there exists $C_{\varepsilon}>0$ such that

$$
\left|\int_{\Omega} f(u) u\right| \leq \varepsilon\left\|\partial_{1} u\right\|_{p_{1}}^{p_{1}}+C_{\varepsilon}\|u\|^{\alpha}, \quad \forall u \in X .
$$

Hence

$$
\begin{aligned}
\Phi^{\prime}(u) u & =\sum_{i=1}^{N}\left\|\partial_{i} u\right\|_{p_{i}}^{p_{i}}-\int_{\Omega} f(u) u \geq \sum_{i=1}^{N}\left\|\partial_{i} u\right\|_{p_{i}}^{p_{i}}-\varepsilon\left\|\partial_{1} u\right\|_{p_{1}}^{p_{1}}-C_{\varepsilon}\|u\|^{\alpha} \\
& \geq(1-\varepsilon) \sum_{i=1}^{N}\left\|\partial_{i} u\right\|_{p_{i}}^{p_{i}}-C_{\varepsilon}\|u\|^{\alpha} \geq C(1-\varepsilon)\|u\|^{p_{N}}-C_{\varepsilon}\|u\|^{\alpha} .
\end{aligned}
$$

Taking $\varepsilon<1$ we get

$$
\liminf _{u \rightarrow 0} \frac{\Phi^{\prime}(u) u}{\|u\|^{p_{N}}}>0
$$

Note also that

$$
\Phi(u)=I_{0}(u)-I(u) \quad \text { and } \quad \Phi^{\prime}(u) u=J_{0}(u)-J(u),
$$

where

$$
I_{0}(u)=\sum_{i=1}^{N} \frac{1}{p_{i}}\left\|\partial_{i} u\right\|_{p_{i}}^{p_{i}}, \quad I(u)=\int_{\Omega} F(u),
$$




$$
J_{0}(u)=\sum_{i=1}^{N}\left\|\partial_{i} u\right\|_{p_{i}}^{p_{i}} \text { and } \quad J(u)=\int_{\Omega} f(u) u
$$

From the compact embedding $X \subset L^{\alpha}(\Omega)$, it follows that $I$ and $J$ are weakly continuous. Moreover there exists $C>0$ such that

$$
I_{0}(t u) \geq \frac{t^{p_{1}}}{p_{N}} \sum_{i=1}^{N}\left\|\partial_{i} u\right\|_{p_{i}}^{p_{i}} \geq C \frac{t^{p_{1}}}{p_{N}}\|u\|^{p_{N}}
$$

if $t>1$ and $\|u\| \leq 1$. In particular, if $u \in \mathcal{S}$ then $I_{0}(t u) \rightarrow \infty$ as $t \rightarrow \infty$. In addition, it is clear that $J_{0}\left(u_{n}\right) \rightarrow 0$ if and only if $u_{n} \rightarrow 0$ in $X$. From (2) we have

$$
\frac{\Phi(t u)}{t^{p_{N}}}=\sum_{i=1}^{N} \frac{t^{p_{i}-p_{N}}}{p_{i}}\left\|\partial_{i} u\right\|_{p_{i}}^{p_{i}}-\int_{\Omega} \frac{F(t u)}{t^{p_{N}}} \rightarrow-\infty
$$

whereas, from (4), it follows that

$$
t \mapsto t^{1-p_{N}} \Phi^{\prime}(t u) u=\sum_{i=1}^{N} t^{p_{i}-p_{N}}\left\|\partial_{i} u\right\|_{p_{i}}^{p_{i}}-\int_{\Omega} \frac{f(t u)}{t^{p_{N}-1}} \text { is decreasing in }(0, \infty) .
$$

Still by (4), we have that

$$
t \mapsto \frac{1}{p_{N}} f(t) t-F(t) \text { is increasing in }(0, \infty) .
$$

Thus for any $u \neq 0$

$t \mapsto \Phi(t u)-\frac{1}{p_{N}} \Phi^{\prime}(t u) t u=\sum_{i=1}^{N}\left(\frac{1}{p_{i}}-\frac{1}{p_{N}}\right) t^{p_{i}-p_{N}}\left\|\partial_{i} u\right\|_{p_{i}}^{p_{i}}+\int_{\Omega}\left(\frac{1}{p_{N}} f(t u) t-F(t u)\right)$ is increasing in $(0, \infty)$.

Finally, it is clear that $u \mapsto \Phi^{\prime}(u) u$ and $\mapsto \Phi(u)-\frac{1}{p_{N}} \Phi^{\prime}(u) u$ are weakly lower semicontinuous on $X$, and $\Phi$ is even.

In view of Theorem 1.2 and Remark 1.1 (1), we infer that (1.7) has a nontrivial and non-negative ground state.

Corollary 2.6. Under the assumptions of Corollary [2.5, the problem (1.7) has infinitely many pairs of solutions.

Proof. Let $\left(u_{n}\right) \subset \mathcal{N}$ be a Palais-Smale sequence for $\Phi$, defined in (2.16). Since $\left(u_{n}\right)$ is bounded, passing to a subsequence, if necessary, we have

$u_{n} \rightarrow u$ in $D_{0}^{1, \vec{p}}(\Omega), u_{n} \rightarrow u$ in $L^{\sigma}(\Omega)$ for $\sigma \in\left[1, p^{*}\right)$, and $u_{n} \rightarrow u$ a.e in $\Omega$.

Since $|f(t)| \leq C\left(1+|t|^{\alpha-1}\right)$ for every $t \in \mathbb{R}$, we get

$$
\int_{\Omega} f\left(u_{n}\right) u_{n}-\int_{\Omega} f\left(u_{n}\right) u=o(1) .
$$

Now, from $u_{n} \rightarrow u$ in $D_{0}^{1, \vec{p}}(\Omega)$ we have

$$
\sum_{i=1}^{N} \int_{\Omega}\left|\partial_{i} u\right|^{p_{i}-2} \partial_{i} u \partial_{i} u_{n}-\sum_{i=1}^{N} \int_{\Omega}\left|\partial_{i} u\right|^{p_{i}}=o(1) .
$$


We use now the standard inequality (cf. 29])

$$
\left(|x|^{p-2} x-|y|^{p-2} y\right)(x-y) \geq \begin{cases}C_{p}|x-y|^{p}, & \text { if } p \geq 2 \\ C_{p} \frac{|x-y|^{2}}{(|x|+|y|)^{2-p}}, & \text { if } 1<p<2,\end{cases}
$$

which holds for some $C_{p}>0$. Note that if $1<p_{i}<2$ then

$$
\begin{aligned}
\left\|\partial_{i} u_{n}-\partial_{i} u\right\|_{p_{i}}^{p_{i}} & =\int_{\Omega} \frac{\left|\partial_{i} u_{n}-\partial_{i} u\right|^{p_{i}}}{\left(\left|\partial_{i} u_{n}\right|+\left|\partial_{i} u\right|\right)^{\frac{p_{i}\left(2-p_{i}\right)}{2}}}\left(\left|\partial_{i} u_{n}\right|+\left|\partial_{i} u\right|\right)^{\frac{p_{i}\left(2-p_{i}\right)}{2}} \\
& \leq\left(\int_{\Omega} \frac{\left|\partial_{i} u_{n}-\partial_{i} u\right|^{2}}{\left(\left|\partial_{i} u_{n}\right|+\left|\partial_{i} u\right|\right)^{2-p_{i}}}\right)^{\frac{p_{i}}{2}}\left(\int_{\Omega}\left(\left|\partial_{i} u_{n}\right|+\left|\partial_{i} u\right|\right)^{p_{i}}\right)^{\frac{2-p_{i}}{2}} \\
& \leq C\left(\int_{\Omega}\left(\left|\partial_{i} u_{n}\right|^{p_{i}-2} \partial_{i} u_{n}-\left|\partial_{i} u\right|^{p_{i}-2} \partial_{i} u\right)\left(\partial_{i} u_{n}-\partial_{i} u\right)\right)^{\frac{p_{i}}{2}}
\end{aligned}
$$

Thus

$$
\int_{\Omega}\left(\left|\partial_{i} u_{n}\right|^{p_{i}-2} \partial_{i} u_{n}-\left|\partial_{i} u\right|^{p_{i}-2} \partial_{i} u\right)\left(\partial_{i} u_{n}-\partial_{i} u\right) \geq \begin{cases}C\left\|\partial_{i} u_{n}-\partial_{i} u\right\|_{p_{i}}^{p_{i}}, & \text { if } p_{i} \geq 2 \\ C\left\|\partial_{i} u_{n}-\partial_{i} u\right\|_{p_{i}}^{2}, & \text { if } 1<p_{i}<2 .\end{cases}
$$

for some $C>0$, and consequently

$$
\begin{aligned}
\Phi^{\prime}\left(u_{n}\right)\left(u_{n}-u\right) & =\sum_{i=1}^{N} \int_{\Omega}\left|\partial_{i} u_{n}\right|^{p_{i}-2} \partial_{i} u_{n}\left(\partial_{i} u_{n}-\partial_{i} u\right)+o(1) \\
& =\sum_{i=1}^{N} \int_{\Omega}\left(\left|\partial_{i} u_{n}\right|^{p_{i}-2} \partial_{i} u_{n}-\left|\partial_{i} u\right|^{p_{i}-2} \partial_{i} u\right)\left(\partial_{i} u_{n}-\partial_{i} u\right)+o(1) \\
& \geq C\left(\sum_{p_{i} \geq 2}\left\|\partial_{i} u_{n}-\partial_{i} u\right\|_{p_{i}}^{p_{i}}+\sum_{1<p_{i}<2}\left\|\partial_{i} u_{n}-\partial_{i} u\right\|_{p_{i}}^{2}\right)+o(1)
\end{aligned}
$$

Therefore $\left\|\partial_{i} u_{n}-\partial_{i} u\right\|_{p_{i}} \rightarrow 0$ for $i=1, \ldots, N$, so that $u_{n} \rightarrow u$ in $D_{0}^{1, \vec{p}}(\Omega)$ and the proof is complete.

\section{REFERENCES}

[1] Adimurthi, Existence of positive solutions of the semilinear Dirichlet problem with critical growth for the n-Laplacian. Ann. Scuola Norm. Sup. Pisa Cl. Sci. (4) 17 (1990), no. 3, 393413.

[2] C. O. Alves, F. J. S. A. Corra, G. M. Figueiredo, On a class of nonlocal elliptic problems with critical growth. Differ. Equ. Appl. 2 (2010), no. 3, 409-417.

[3] C. O. Alves, A. El Hamidi, Existence of solution for a anisotropic equation with critical exponent, Differential Integral Equations, 21 (2008), 25-40.

[4] A. Ambrosetti and E. Colorado, Standing waves of some coupled nonlinear Schrdinger equations, J. Lond. Math. Soc., 75 (2007), 67-82.

[5] M. Badiale, E. Serra, Semilinear elliptic equations for beginners. Existence results via the variational approach. Universitext. Springer, London, 2011.

[6] A. Bahri and H. Berestycki, A perturbation method in critical point theory and applications, Trans. Amer. Math. Soc. 267 (1981), 1-32.

[7] T. Bartsch, Z.-Q. Wang, Zhi-Qiang, M. Willem, The Dirichlet problem for superlinear elliptic equations. Stationary partial differential equations. Vol. II, 155, Handb. Differ. Equ., Elsevier/North-Holland, Amsterdam, 2005.

[8] C. J. Batkam, Ground state solution of a nonlocal boundary-value problem. Electron. J. Differential Equations 2013, No. 257, 8 pp.

[9] V. Benci, A.M. Micheletti and D. Visetti, An eigenvalue problem for a quasilinear elliptic field equation, J. Differential Equations 184 (2002), 299-320. 
[10] P. A. Binding, P. Drabek, Y. X, Huang, On Neumann boundary value problems for some quasilinear elliptic equations, Vol. 1997(1997), No. 05. pp. 1-11.

[11] K.J. Brown, Y. Zhang, The Nehari manifold for a semilinear elliptic equation with a signchanging weight function. J. Differential Equations 193 (2003), no. 2, 481-499.

[12] K.J. Brown, T-F. Wu, A fibering map approach to a semilinear elliptic boundary value problem. Electron. J. Differential Equations 2007, No. 69, 9 pp. (electronic)

[13] A. Castro, J. Cossio and J. M. Neuberger, A sign-changing solution for a superlinear Dirichlet problem, Rocky Mountain J. Math. 27 (1997), 1041-1053.

[14] L. Cherfils and Y. Ilyasov, On the stationary solutions of generalized reaction diffusion equations with $p \& q$-Laplacian, Commun. Pure Appl. Anal. 4 (2005), no. 1, 9-22.

[15] S. Cingolani, M. Degiovanni, Nontrivial solutions for $p$-Laplace equations with right-hand side having $p$-linear growth at infinity, Comm. Partial Differential Equations 30 (2005), no. 7-9, 1191-1203.

[16] C. V. Coffman and W. K. Ziemer, A prescribed mean curvature problem on domains without radial symmetry, SIAM J. Math. Anal. 22(4), 982-990 (1991).

[17] A. Di Castro, Existence and Regularity Results for Anisotropic Elliptic Problems, Adv. Nonlinear Stud., 9 (2009), 367-393.

[18] J.M. Do Ó, P. Ubilla, A multiplicity result for a class of superquadratic Hamiltonian systems, Electron. J. Differential Equations 2003 (15) (2003) 114.

[19] G. M. Figueiredo, Existence of a positive solution for a Kirchhoff problem type with critical growth via truncation argument. J. Math. Anal. Appl. 401 (2013), no. 2, 706-713.

[20] G. M. Figueiredo, Existence of positive solutions for a class of $p \& q$ elliptic problems with critical growth on $R^{N}$, J. Math. Anal. Appl. 378 (2011), no. 2, 507-518

[21] I. Fragalà, F. Gazzola, B. Kawohl, Existence and nonexistence results for anisotropic quasilinear elliptic equations. Ann. Inst. H. Poincar Anal. Non Linaire 21 (2004), no. 5, 715-734.

[22] L. Gasinski, N. S. Papageorgiou, Anisotropic nonlinear Neumann problems. Calc. Var. Partial Differential Equations 42 (2011), no. 3-4, 323354.

[23] X. He, W. Zou, Ground states for nonlinear Kirchhoff equations with critical growth. Ann. Mat. Pura Appl. (4) 193 (2014), no. 2, 473-500.

[24] S.Hu, N.S. Papageorgiou, Solutions of nonlinear nonhomogeneous Neumann and Dirichlet problems. Commun. Pure Appl. Anal. 12 (2013), no. 6, 2889-2922.

[25] T. F. Ma, Remarks on an elliptic equation of Kirchhoff type, Nonlinear Anal., 63 (2005), e1967-e1977.

[26] D. Mugnai, N. S. Papageorgiou, Wang's multiplicity result for superlinear (p,q)-equations without the Ambrosetti-Rabinowitz condition. Trans. Amer. Math. Soc. 366 (2014), no. 9, 4919-4937.

[27] D. Naimen, The critical problem of Kirchhoff type elliptic equations in dimension four. J. Differential Equations 257 (2014), no. 4, 1168-1193.

[28] Z. Nehari, On a class of nonlinear second-order differential equations, Trans. Amer. Math. Soc. 95 (1960), 101-123.

[29] J. Simon, Regularité de la solution dune equation non lineaire dans $\mathbb{R}^{N}$, Lecture Notes in Math. No. 665 P. Benilan editors Springer Verlag, 1978.

[30] A. Szulkin and T. Weth, The method of Nehari manifold. Handbook of nonconvex analysis and applications, 597-632, Int. Press, Somerville, MA, 2010.

[31] G. Tarantello, On nonhomogeneous elliptic equations involving critical Sobolev exponent. Ann. Inst. H. Poincaré Anal. Non Linéaire 9 (1992), no. 3, 281-304.

[32] J. Vétois, Existence and regularity for critical anisotropic equations with critical directions, Adv. Differential Equations, 16 (2011), 61-83.

Giovany M. Figueiredo

Universidade Federal do Pará, Faculdade de Matemática, 66075-110, Belém-PA, Brazil E-mail address: giovany@ufpa.br

H. RAMOS QUOIRIN

Universidad de Santiago de Chile, Casilla 307, Correo 2, Santiago, Chile

E-mail address: humberto.ramos@usach.cl 